\title{
PÉRDIDAS DE PRESIÓN DEL FLUJO DE AIRE POR PILAS DE MADERA
}

\author{
RODOLFO NEUMANN R., ALBERTO TAMM R. Y MAURICIO BRAVO H.
}

Depto. de Ingenieria Mecánica, Universidad de Concepción. Casilla 53-C, Concepción, Chile

\section{RESUMEN}

Se determinaron las pérdidas de presión del flujo de aire que pasa por una pila de madera en un amplio rango de condiciones de operación que se presentan en secadores comerciales.

Se realizaron ensayos en un tinel de viento ubicando tablas de $40 \times 200 \times 460 \mathrm{~mm}$ en forma transversal al flujo. Las tablas se apilaron dejando distancias verticales de 15, 20 y $25 \mathrm{~mm}$. y distancias horizontales entre 0 y $50 \mathrm{~mm}$. La velocidad del aire en las rendijas se varió entre 0,5 $y 10 \mathrm{~m} / \mathrm{s}$.

Se determinó que la pérdida de presión a través de la pila depende tanto de la separación vertical entre las tablas como de la distancia horizontal que las separa, cuando se utilizan separadores de $20 \mathrm{~mm}$ o menos. Con rendijas de mayor altura los efectos de las separaciones horizontales son irrelevantes. Se observó que la transición entre los regimenes laminar y turbulento se produce con velocidades entre 1.2 y $1,8 \mathrm{~m} / \mathrm{s}$. Se presenta una correlación que permite calcular el factor de fricción regular en función del número de Reynolds y de la separación vertical entre las tablas. Con humo se pudo observar la formación de torbellinos en el borde que enfrenta el flujo y entre las tablas. Los resultados pueden ser usados para mejorar el cálculo y la operación de secadores de madera.

Palabras claves: Secado, Ventilación, Velocidad.

\section{ABSTRACT}

Pressure drops of the air flow' through stacks of boards were determined in a wide range of operating conditions in commercial timber drying kilns.

For the experiments $50 \times 200 \times 460 \mathrm{~mm}$ boards were placed in a test section of a wind tunnel perpendicular to the air flow. The boards were stacked with vertical separations of 15,20 and $25 \mathrm{~mm}$ and horizontal separations of 0 to $50 \mathrm{~mm}$. The air velocity was adjusted from 0.5 to 10 $m / s$.

It was concluded that the pressure drop through a stack depends on the vertical distance of the boards and also on the horizontal distance which separates them, when $20 \mathrm{~mm}$ or thinner stickers are used. When the gaps between boards are higher horizontal spaaces between boards do not affect pressure drop. It was observed that the transition of laminar to turbulent flow occurs at velocities ranging between 1.2 and $1.8 \mathrm{~m} / \mathrm{s}$. A correlation is presented to calculate the friction factor as a function of the Reynolds number and the vertical distance between boards. With fume the onset of turbulence at the edge and between the boards could be observed. The results may be used to improve calculations and operation of drying kilns.

Keywords: Kiln Drying. Air Flow, Velocity 


\section{INTRODUCCIÓN}

Durante el secado artificial se impulsa aire caliente a través de pilas de madera para aportar el calor de evaporación y retirar la humedad de las tablas. Según la rapidez de secado, el ancho de las pilas y otros parámetros, la velocidad del aire entre las tablas puede variar entre 0,5 y $8 \mathrm{~m} / \mathrm{s}$. Mientras mayor sea ésta, mayor será el coeficiente de transferencia de calor entre el aire y las tablas y menor será el grado de saturación del aire al final de la rendija, lo que aumenta la tasa de secado. Como en general los costos de inversión y operación de un secador dependen fuertemente de su capacidad de ventilación. también es necesario conocer las pérdidas de presión que sufre el aire al pasar a través de las pilas.

En la literatura especializada se encuentran pocos trabajos que estudian el problema planteado. Sorensen (1969) concluyó que se forma una capa laminar detrás de un torbellino estacionario en el canto de la tabla que enfrenta el flujo, porque parte de éste debe cambiar de dirección gradualmente, como se observa en la Figura $\mathrm{N}^{\circ} 1$. Además indica que existe un área turbulenta sobre la capa limite. causada por el desprendimiento de torbellinos desde el torbellino estacionario. En su trabajo también presenta correlaciones que permiten calcular coeficientes de transferencia de masa. Langrish et al. (1992) observaron que la separación horizontal entre tablas genera una turbulencia adicional en comparación con la placa plana. Esta turbulencia sería la causa de un aumento de los coeficientes de transferencia de masa en esta zona. Además observaron la generación de torbellinos entre las tablas y la existencia de torbellinos deformados a lo largo de la superficie de la tabla.

\section{Zona de torbellinos}

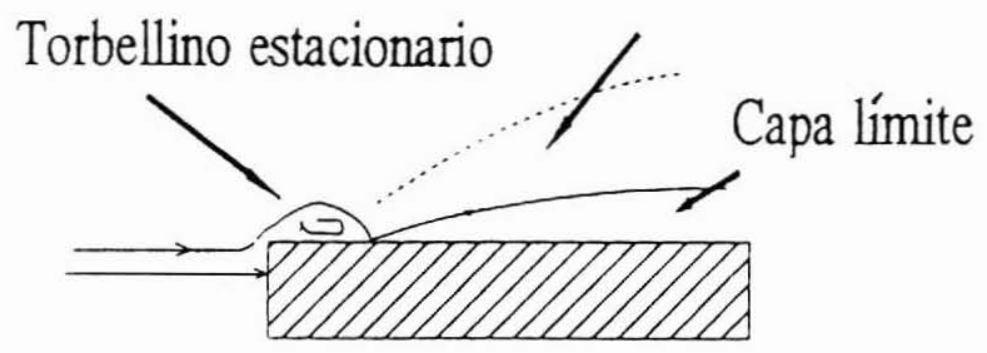

Figura No 1 . INVESTIGACIÓN DE SORENSEN 
Brunner (1989) menciona la importancia de la velocidad del aire, indicando que la zona crítica entre los escurrimientos laminar y turbulento se encuentra entre 0,5 y 1,5 $\mathrm{m} / \mathrm{s}$. Indica que con $0,5 \mathrm{~m} / \mathrm{s}$ se dobla el tiempo de secado en relación a $1,5 \mathrm{~m} / \mathrm{s}$. Menciona que el tiempo de secado prácticamente no es afectado por el hecho de separar horizontalmente las tablas.

De la ecuación de la energía para flujos isocóricos, turbulentos, en régimen permanece y por conductos de sección transversal constante, se obtiene la ecuación de Darcy-Weisbach. La pérdida de presión es:

$$
\Delta p=f \frac{L}{D} \rho \frac{V^{2}}{2}
$$

Aqui $f$ es el factor de fricción. $L$ el largo del conducto (rendija), $D$ el diámetro. $p$ la densidad y $l$ la velocidad del fluido. El factor de fricción $f$ depende del número de Reynolds. $R e=l D \rho / \mu$ y de la razón $\varepsilon \mathrm{D}$. en que $\varepsilon$ es la rugosidad relativa.

$$
f=f\left(\operatorname{Re}, \frac{\varepsilon}{D}\right)
$$

Cuando los conductos no son circulares se pueden aplicar las ecuaciones anteriores utilizando el diámetro hidráulico. Hucbscher (citado en ASHRAE Fundamentals. 1989) presenta la siguiente ecuación para determinar los diámetros equivalentes de ductos rectangulares. $\mathrm{D}_{E}$, que permiten calcular los factores de fricción con diagramas y correlaciones desarrollados para secciones circulares:

$$
D_{E}=1,3 \frac{(\text { ancho } * \text { altura })^{0.625}}{(\text { ancho } * \text { altura })^{0.25}}
$$

Se concluye que según Huebscher el diámefro equivalente es aproximadamente igual al doble del diámetro hidráulico.

Las pérdidas de presión que se presentan en la entrada y en la salida de la pila de madera. se originan por la separación de la corriente del contorno y se pueden calcular como:

$$
\Delta p=K \rho \frac{V^{2}}{2}
$$

Aquí $K$ es el coeficiente de fricción (singular). La pérdida de presión a la salida de la pila de madera se puede calcular como la pérdida producida en una expansión brusca. aplicando las ecuaciones de continuidad y de cantidad de movimiento. considerando la razón del área de la rendija y el área del espacio después de la pila $\left(\mathrm{A}_{1}\right.$ 
$/ A_{2}$ ) como la razón entre el espesor del separador y el de una tabla más el del separador, se obtiene la pérdida de presión:

$$
\Delta p_{E}=K_{E} \rho \frac{V^{2}}{2} \quad K_{E}=\left[1-\left(\frac{A_{1}}{A_{2}}\right)^{2}\right]^{2} \approx 0,79
$$

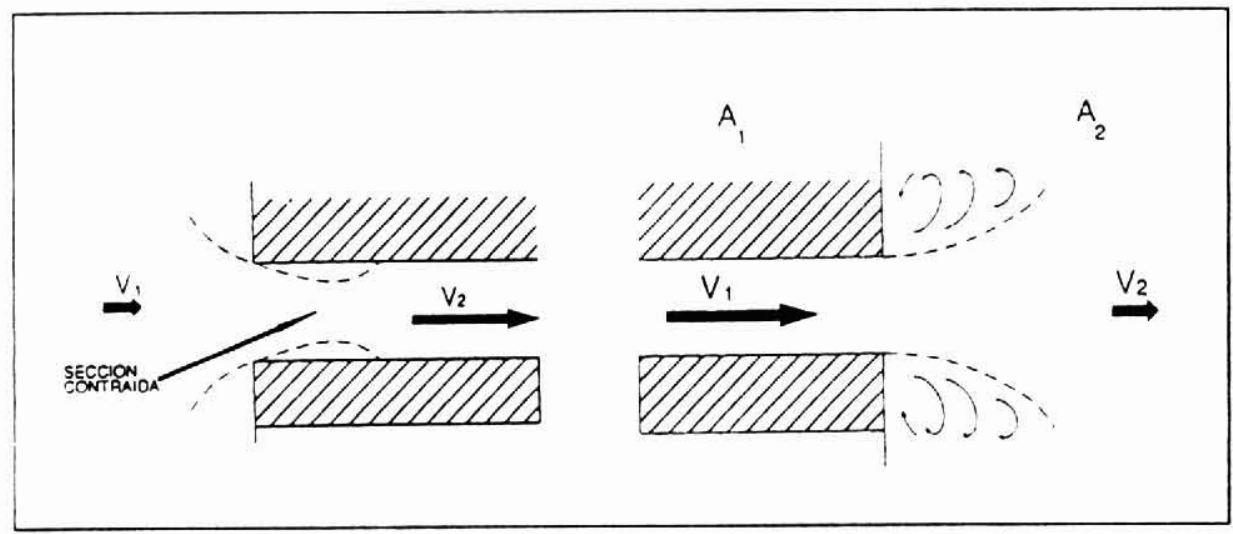

Figura No2. CONTRACCIÓN Y EXPANSIÓN BRUSCA

La entrada del aire a la pila de madera se puede considerar como una contracción brusca, en la cual la corriente se separa de la pared después del borde. La sección contraida se forma debido a que el aire que fluye sobre el borde exterior del escurrimiento no puede doblar en ángulo recto con depresiones finitas. El proceso de convertir la presión estática en presión dinámica es muy eficiente, por ello en la entrada a la sección contraída la pérdida de carga es pequeña comparada con la pérdida de carga que ocurre después de la sección contraída, donde la presión dinámica es reconvertida en presión estática. Aplicando las ecuaciones de continuidad, cantidad de movimiento y el factor de contracción. que depende de la geometria y del número de Revnolds, se obtiene:

$$
\Delta p_{c}=K_{c} \rho \frac{V^{2}}{2} \quad K_{c}=0,5
$$




\section{METODOLOGIA}

Para realizar las mediciones experimentales se agregó una sección rectangular de $310 \mathrm{~mm}$ de altura, $490 \mathrm{~mm}$ de ancho y $2.900 \mathrm{~mm}$ de largo a un túnel de viento existente. Las paredes laterales tenian ventanas de acrílico. Las tablas no estaban cepilladas y tenían $40 \mathrm{~mm}$ de espesor, $200 \mathrm{~mm}$ de ancho y $460 \mathrm{~mm}$ de largo. Las tablas se apoyaron en perfiles $\mathrm{L}$, sujetos a las paredes laterales, y se podian ubicar en alturas diferentes.

La pérdida de presión del flujo de aire a través de la pila se determinó colocando cañerias de cobre en forma normal al flujo y a una distancia de $2.480 \mathrm{~mm}$. La señal se midió con un manómetro diferencial digital, marca Modus con un rango de $\pm 100 \mathrm{~Pa}$ y una sensibilidad de $1 \mathrm{~Pa}$.

La velocidad fue medida con un anemómetro de hilo caliente digital, marca TriSence de Cole Parmer, modelo 3700 . rango de $0,03 \mathrm{~m} / \mathrm{s}$ a $25 \mathrm{~m} / \mathrm{s}$, resolución de 0,01 $\mathrm{m} / \mathrm{s}$ y error $\pm 3 \%$ de lectura. El hilo caliente está ubicado en la punta de una varilla, que fue instalada en el centro de una de las rendijas de la pila de madera (Figura $\mathbf{N}^{\circ} 3$ ).

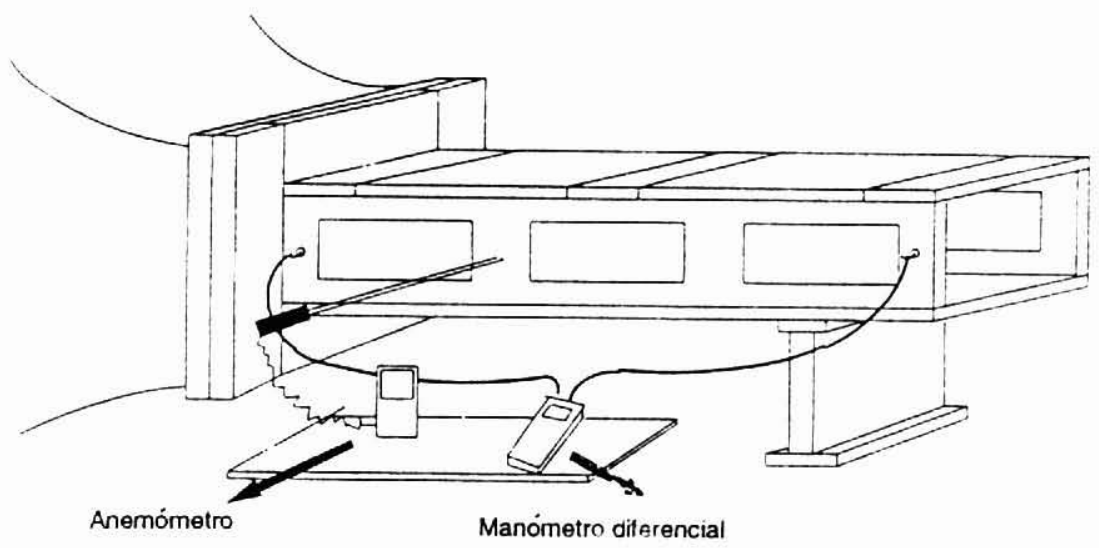

Figura $N^{\circ} 3$. BANCO DE ENSAYOS

Para determinar los perfiles de velocidad para varias velocidades, alturas y posiciones de la tabla en una rendija, se construyó un dispositivo que permitía ubicar un tubo pitot diferencial a lo largo y alto de ésta. 
Para observar el escurrimiento se inyectó humo blanco en la entrada de una rendija de la pila. Este fue producido haciendo burbujear vapor de amoníaco en ácido clorhídrico. Utilizando una buena iluminación se pudo filmar y fotografiar el escurrimiento.

En los ensayos se variaron los siguientes parámetros, que en parte se explican en la Figura $\mathrm{N}^{\circ} 4$.
Velocidad del aire
$0-10 \mathrm{~m} / \mathrm{s}$

Separación horizontal entre tablas

(en el sentido del escurrimiento) : $0,10,20,50 \mathrm{~mm}$.

Separación vertical entre tablas : : $15,20,25 \mathrm{~mm}$.



Separación entre tablas ( Sep.)

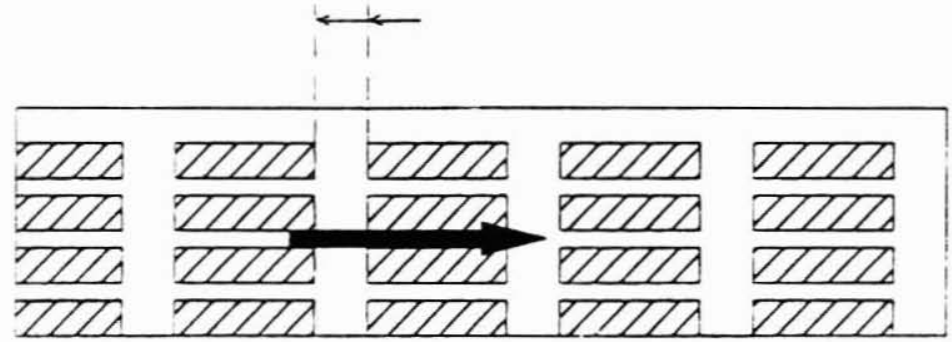

Figura $N^{\circ} 4$. APILAMIENTO DE LAS TABLAS

Los factores de fricción asociados a la pérdida de presión regular se determinaron realizando experimentos con tablas juntas. esto es sin separación horizontal. 
Posteriormente. se determinaron las pérdidas singulares colocando las tablas a $10,20 \mathrm{y}$ $50 \mathrm{~mm}$ de distancia horizontal de la siguiente forma:

$$
\Delta p_{T}=\Delta p_{R}+\Delta p_{S}
$$

Aqui $\Delta p_{T}$ es la pérdida de presión total medida, $\Delta p_{R}$ es la pérdida regular obtenida del ensayo sin separación horizontal y $\Delta p_{S}$ es la pérdida de presión singular debido a la separación horizontal entre las tablas. De esta forma se obtiene para los ensayos con separación horizontal:

$$
\Delta p_{T}=f \frac{L}{D} \rho \frac{V^{2}}{2}+\varepsilon \rho \frac{V^{2}}{2}
$$

En esta ecuación se puede despejar el coeficiente de pérdida de presión singular:

$$
\varepsilon=\frac{2 \Delta p_{T}}{\rho V^{2}}-f \frac{L}{D}
$$

Para obtener la pérdida de carga singular por espacio se divide el coeficiente de pérdida de presión singular $\varepsilon$ calculado anteriormente por el número de separaciones horizontales. El valor de $\varepsilon$ solo tiene validez para la zona turbulenta, porque es en esta zona donde la pérdida de presión varía con el cuadrado de la velocidad, que es la forma en que se formuló la pérdida de presión total.

\section{RESULTADOS Y DISCUSIÓN}

\section{Pérdida de Carga a Través de la Pila de Madera}

Como era de esperar, se puede observar en la Figura №5 que la diferencia de presión aumenta exponencialmente con la velocidad y disminuye con el aumento de la separación vertical entre las tablas. A $2 \mathrm{~m} / \mathrm{s}$ la pérdida de presión es inferior a $4 \mathrm{~Pa} / \mathrm{m}$, mientras que a $5 \mathrm{~m} / \mathrm{s}$ este valor puede llegar a $25 \mathrm{~Pa} / \mathrm{m}$ con una distancia vertical de 15 $\mathrm{mm}$.

Con una separación vertical entre tablas de $15 \mathrm{~mm}$, las separaciones horizontales afectaron la pérdida de carga por igual en la medida que éstas eran iguales o superiores a $10 \mathrm{~mm}$. Con una separación vertical de $20 \mathrm{~mm}$, que es lo más común en la práctica industrial, la pérdida de carga aumenta significativamente con la separación horizontal. Al estar las tablas separadas en la altura en $25 \mathrm{~mm}$ las diferencias causadas por no juntar las tablas en un plano horizontal son irrelevantes. 

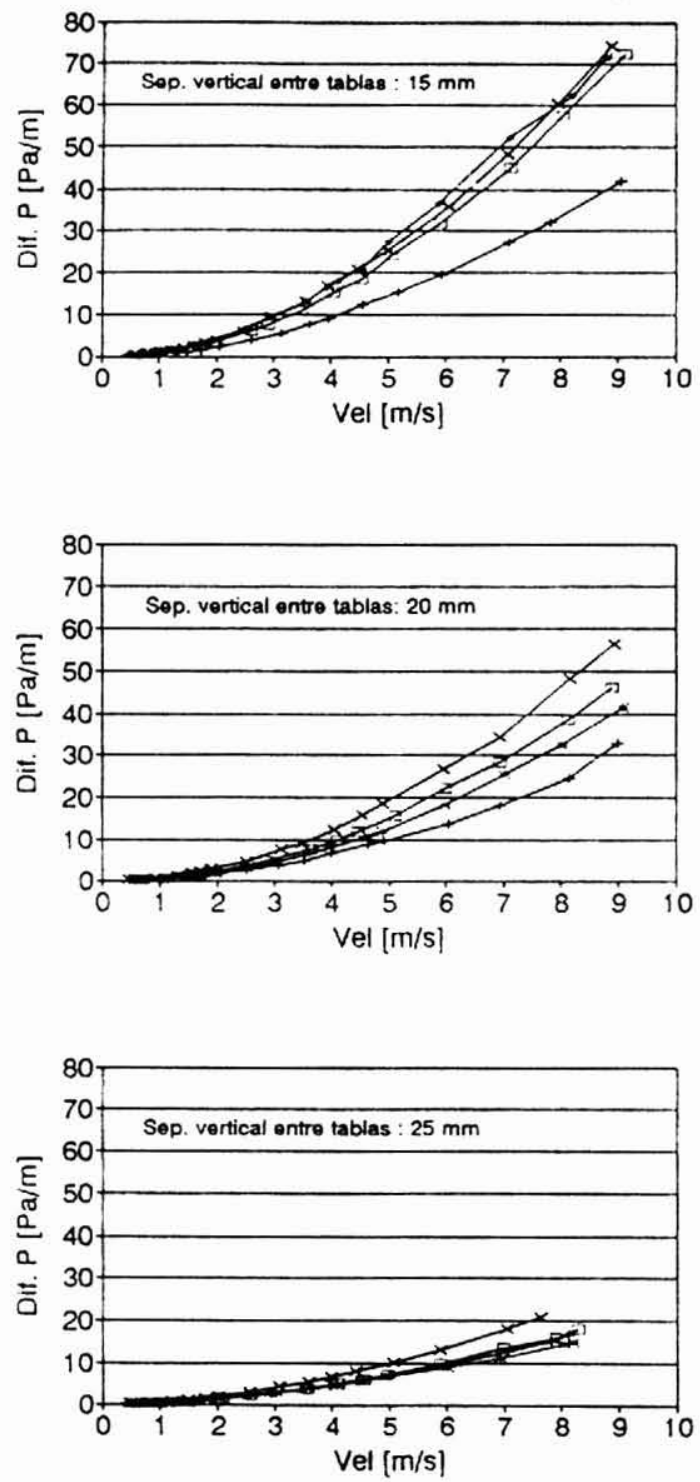

$\rightarrow$ Sep $0 \mathrm{~mm} . \longrightarrow$ Sep. $10 \mathrm{~mm} . \rightarrow$ Sep. $20 \mathrm{~mm} . \rightarrow$ Sep. $50 \mathrm{~mm}$

Figura N ${ }^{\circ} 5$. DIFERENCIA DE PRESIÓN V/S VELOCIDAD 


\section{Factores de Fricción}

En la Figura $N^{\circ} 6$ se distingue el rango de velocidades para el cual el escurrimiento es laminar, la zona de transición y la zona donde es turbulento. Esto es válido para los casos con separación vertical entre tablas de 15 y $20 \mathrm{~mm}$, encontrándose la zona de transición entre 1 y $1.8 \mathrm{~m} / \mathrm{s}$. Para el ensayo con separación vertical de $25 \mathrm{~mm}$. no se observa claramente esta zona.

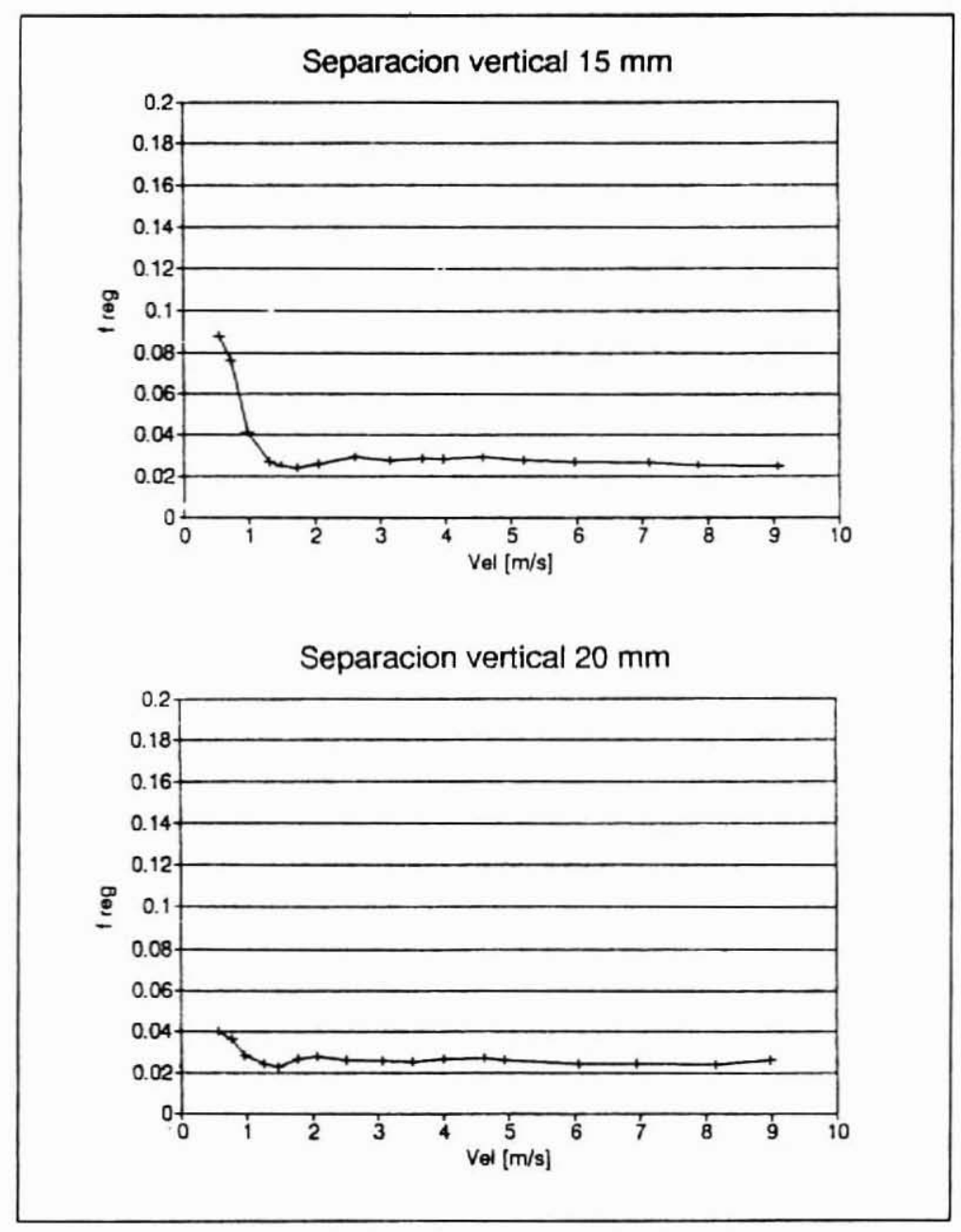

Figura N6. FACTOR DE FRICCIÓN REGULAR 
Con los resultados del cálculo del factor de pérdida de presión regular $f$, se obtuvo una correlación en función del número de Reynolds $\operatorname{Re}_{\mathrm{D}} \mathrm{y}$ de la separación vertical entre tablas $D$. La correlación tiene validez en la zona turbulenta, debido a que al determinar el factor $f$ se consideró un escurrimiento de este tipo. Las otras correlaciones usadas para determinar este factor, como las de Filolenko y Colebrook, consideran que $f$ varía con el cuadrado de la velocidad, en cambio en la correlación encontrada se observa que sólo varía con un exponente 1,5 .

$$
f_{\text {reg }}=0,25\left(\log R e_{D_{\text {hidr }}}\right)^{1.5}-0,2858 D
$$

Para los ensayos con una separación vertical de $25 \mathrm{~mm}$ la separación horizontal entre tablas no afecta la diferencia de presión. Como los coeficientes de fricción singulares se obtienen de restas (ecuación 9) y éstas son similares a los errores cometidos en la medición, no tienen sentido calcular los coeficientes de fricción según esta metodologia.

Para los ensayos con separación vertical entre tablas de 15 y $20 \mathrm{~mm}$, los coeficientes de pérdida de presión singulares calculados son significativos y por lo tanto razonables, como se muestra en el Cuadro $\mathrm{N}^{\circ} 1$.

Cuadro $\mathrm{N}^{\circ} 1$

COEFICIENTE DE FRICCIÓN SINGULAR

\begin{tabular}{|l|c|c|c|}
\hline & Sep. horizontal $10 \mathrm{~mm}$ & Sep. horizontal $20 \mathrm{~mm}$ & Sep. horizontal $50 \mathrm{~mm}$ \\
\hline Sep. vertical $15 \mathrm{~mm}$ & $\approx 0,19$ & $\approx 0,15$ & $\approx 0,26$ \\
\hline Sep. vertical $20 \mathrm{~mm}$ & $\approx 0,04$ & $\approx 0.07$ & $\approx 0,18$ \\
\hline
\end{tabular}

Para los ensayos con una distancia vertical entre tablas de $15 \mathrm{~mm}$. la separación horizontal prácticamente no afecta el valor de $\varepsilon$, siendo éste aproximadamente 0,2 . En los ensayos en que la separación vertical entre tablas es de $20 \mathrm{~mm}$ la separación entre ellas es aproximadamente proporcional al factor $\varepsilon$.

\section{Perfiles de Velocidades en la Altura de la Rendija}

Se midieron perfiles de velocidades en rendijas de $20 \mathrm{~mm}$ de altura y con una velocidad de $2 \mathrm{~m} / \mathrm{s}$. Al estar las tablas juntas. esto es sin separación horizontal, se observó que el perfil de velocidades es afectada por cada borde de las tablas y no se observó la presencia de torbellinos iniciales.

Con separaciones horizontales de $50 \mathrm{~mm}$. se observó que el perfil de velocidades se desarrolla en cada tabla. por lo que el flujo por una pila de madera no se puede 
considerar como por una rendija plana. También se observó que a $5 \mathrm{~mm}$ del borde de la tabla que enfrenta el flujo existía un torbellino de aproximadamente $2 \mathbf{~ m m}$. de altura.

\section{Observación del Escurrimiento}

Se aplicó la técnica de visualización descrita anteriormente en ensayos con pilas de tablas que tenían una separación vertical de $20 \mathrm{~mm}$, una separación horizontal de 50 $\mathrm{mm}$ y velocidades entre 0 y $10 \mathrm{~m} / \mathrm{s}$. Para velocidades bajo $1 \mathrm{~m} / \mathrm{s}$ no se apreció una renovación de aire en el espacio libre entre las tablas, similar al escurrimiento sobre una cavidad. Para este rango de velocidades se distingue que el escurrimiento es laminar, apreciándose claramente la forma del perfil y el flujo en capas. Para velocidades sobre $2 \mathrm{~m} / \mathrm{s}$ se observa que el flujo de aire con humo es desordenado y turbulento. De lo observado y del cálculo del factor de fricción regular se puede mencionar que el criterio para establecer si el flujo es laminar o turbulento $(R e>2300)$ es válido para el flujo a través de una pila de madera.

En todo el rango de velocidades no se logra apreciar la formación de torbellinos al comienzo de las tablas.

\section{CONCLUSIONES}

De los ensayos para determinar la pérdida de presión a través de la pila de madera se puede concluir que en general ésta depende de la velocidad, de la separación vertical entre las tablas y de la distancia horizontal que las separa cuando los separadores son de $20 \mathrm{~mm}$. de altura o menores. De los ensayos sin separación horizontal entre tablas se obtuvo una correlación para determinar el factor de fricción regular, que depende del número de Reynolds, $R e_{D}$ y de la distancia vertical entre las tablas, $D$.

$\mathrm{Al}$ aplicar la correlación de Colebrook y el gráfico de Moody se obtienen factores de fricción razonables si se utiliza el diámetro equivalente determinado por Huebscher y una rugosidad de $1 \mathrm{~mm}$. También se obtuvieron valores del coeficiente de fricción singular para distintas separaciones horizontales y verticales con los cuales, junto a los factores de fricción regular, se puede calcular con facilidad la pérdida de presión del flujo que atraviesa una pila de madera.

Se estableció que el escurrimiento del aire a través de la pila de madera se puede caracterizar con el número de Reynolds y que la zona de transición entre los regímenes laminar y turbulento se encuentra aproximadamente entre 1,2 y $1,8 \mathrm{~m} / \mathrm{s}$.

Se observó que con tablas separadas se forman torbellinos estables entre y sobre los bordes de éstas. También se forma una nueva capa límite en cada borde. Con tablas juntas el perfil de velocidades también se altera. No se observó el desprendimiento de torbellinos como lo propone Sorensen. 
Estos resultados son de interés en la transferencia de calor y materia, debido a que mediante analogias, como la de Chilton-Colburn, se pueden evaluar los coeficientes de transferencia de calor y materia.

Para efectos prácticos los resultados obtenidos permiten deducir la forma de mejorar la distribución de velocidades en secadores de madera o de aumentar el caudal cuando el sistema de ventilación está subdimensionado.

\section{RECONOCIMIENTOS}

Esta publicación es el resultado de un proyecto de investigación financiado por el Fondo de Ciencia y Tecnología. Los autores agradecen su apoyo y patrocinio.

\section{REFERENCIAS BIBLIOGRÁFICAS}

ASHRAE HANDBOOK, Fundamentals, 1989. 1791 Tullie Circle, N. E. Atlanta, GD 30326.

Streeter, Victor L and Wylie, E. Benjamin, 1987. Mecánica de Fluidos. Editorial Mc. Graw Hill, Mexico. $8^{2}$ edición, $3^{3}$ edición en español.

VDI- Wärmeatlas. Verein Deutscher Ingenieure., 1984 Editorial VDI-Verlag GmbH, Dusseldorf. $4^{4}$ edición.

Sorensen, Ansgar., 1969. Mass Transfer Coefficients on Truncated Stabs. Chemical Engineering Science, 24, 1445 - 1460.

T.A.G. Langrish, P.C.S Kho, R. B. Keey, J.C.F. Walker., 1992. Experimental Measurement and Simulation of Local Mass-transfer Coefficients in Timber Kilns. Drying Tecnology, 10(3), 753-781.

G. Arnanud, J. P. Fohr, J. P. Garnier, C. Ricolleau., 1991. Laboratoire d' Etudes des Systèmes Thermiques et Energetiques. 40, Avenue du Recteur Pineau, 86022 POITTIERS Cédex (France). Drying Technology, 9(1), 183 - 200.

Brunner, Reinhard., 1989 Die Turbo-Trocknung, eine neue Dimension in der Holztrocknungstechnik. Holz Zentralblatt, Stuttgart Nr. 50.

Bravo H., Mauricio., 1994. Determinación de Coeficientes Convectivos de Calor y Madera en una Pila de Madera. Depto. Ingenieria Mecánica, Universidad de Concepción.

Tamm R., Alberto., 1994. Estudio y Determinación de la Pérdida de Carga en un Secador de Madera. Depto. Ingeniería Mecánica, Universidad de Concepción. 